\title{
ALTAS CAPACIDADES Y TRASTORNOS ALIMENTARIOS: ¿VULNERABILIDAD O PROTECCIÓN?
}

\author{
GIFTEDNESS AND EATING DISORDERS: \\ VULNERABILITY OR PROTECTION?
}

\author{
Dra. Laura Hernangómez Criado \\ Psicóloga Clínica \\ Unidad de Trastornos Alimentarios. Complejo Hospitalario de Toledo. España \\ ORCID: https://orcid.org/0000-0003-2502-9056
}

Cómo referenciar este artículo/How to reference this article:

Hernangómez Criado, L. (2020). Altas Capacidades y Trastornos Alimentarios: ¿Vulnerabilidad o Protección?. Revista de Psicoterapia, 31(115), 77-95. https://doi.org/10.33898/rdp.v31i115.357

\section{Resumen}

En este artículo, se revisa la relación entre altas capacidades y trastornos alimentarios, explorando los factores que pueden explicar su conexión: asincronías, sensibilidad y perfeccionismo. Profundizando en los procesos por los que pueden dar lugar a la psicopatología, se concluye que es la interacción con un medio poco preparado para responder a las necesidades del menor lo que las convierte en variables de vulnerabilidad y que podrían orientarse de una forma constructiva previniendo ese efecto. La atención a aspectos socioafectivos en el desarrollo, el fomento y valoración de la creatividad y la creación de contextos educativos estimulantes en los que el proceso de aprendizaje sea más importante que un determinado resultado podría impactar de forma positiva en la prevención de los trastornos alimentarios.

Palabras clave: Altas capacidades, Superdotación, Trastornos Alimentarios, Vulnerabilidad, Asincronía, Perfeccionismo, Sensibilidad.

\begin{abstract}
In this article, relationship between giftedness and eating disorders is explored by reviewing the factors which may explain their connection: asynchronies, sensitivity and perfectionism. Provided further knowledge of the processes which may lead to psychopathology, it is concluded that these variables become vulnerability factors because of their interaction with an environment that is poorly responsive to the needs of the gifted child. However, it would be possible to design different settings to prevent this: paying attention to socio-affective development, promotion and appreciation of creativity, and stimulating educational environments in which the learning process is more important than performance goals could have a significant impact on prevention of eating disorders.
\end{abstract}

Keywords: Giftedness, High intellectual capacity, High Ability, Eating Disorders, Vulnerability, Asynchrony, Perfectionism, Sensitivity. 


\section{Introducción}

Los trastornos alimentarios suponen un auténtico reto para la sanidad, no sólo por el elevado impacto a diferentes niveles de salud y funcionamiento (Ágh et al., 2016) sino también por el aumento del número de casos cada vez más rápido en las últimas décadas (Galmiche, Déchelotte, Lambert y Tavolacci, 2019). A pesar de los avances en nuestro conocimiento de estas patologías, necesitamos más luz sobre las variables que contribuyen al riesgo o protección contra estos trastornos $\mathrm{y}$, sobre todo, los procesos por los que dichas variables convierten a una persona en vulnerable ya que sólo de ese modo podremos optimizar las estrategias de intervención terapéutica y preventiva. En ese objetivo se encuadra esta revisión.

La relación entre trastornos alimentarios y alta capacidad cognitiva se señaló pronto tanto desde el ámbito de investigación de los trastornos alimentarios (e.g., Garner, 1991) como desde el estudio de la superdotación (e.g., Detzner y Schmidt, 1986), siendo una tendencia que se ha observado repetidamente en anorexia nerviosa (López, Stahl y Tchanturia, 2010). A pesar de esa asociación demostrada, ha tendido a contemplarse sólo como un dato descriptivo, una observación espuria en el peor de los casos, o un factor de riesgo más en el mejor de los casos, sin profundizar en los mecanismos por los cuales una elevada capacidad cognitiva puede convertirse en factor de vulnerabilidad.

El concepto de altas capacidades se refiere a la excepcionalidad cognitiva en uno o varios dominios e implica un funcionamiento peculiar a nivel de procesos, estrategias, flexibilidad y creatividad (López, 2007). Sin embargo, la operativización del constructo ha hecho que tienda a identificarse con una medida estática y monolítica del cociente intelectual (Borland, 2009), no sólo en detrimento de una comprensión multidimensional que incluiría componentes lógico-deductivos y creativos, sino también del reconocimiento de los procesos diferenciales asociados y su interacción con el resto de áreas de funcionamiento. Las altas capacidades en una persona pueden afectar a su forma de entender el mundo, las relaciones, las emociones y a sí misma, lo que precisamente tiene implicaciones en los diferentes factores de vulnerabilidad para el trastorno alimentario (e.g., Fairburn, Cooper y Shafran, 2003, 2008).

La superdotación intelectual ha sido descrita como "un drama" (Society for the Advancement of Gifted Education, 1998), un potencial ventajoso para extraer el máximo beneficio a un entorno favorable (Pluess y Belsky, 2013) o un factor de resiliencia en circunstancias desfavorables a pesar de la inconsistente evidencia empírica para afirmarlo (Friborg, Barlaug, Martinussen, Rosenvinge y Hjemdal, 2005; Moon, 2009). Y es posible que, paradójicamente, todos tengan razón. Las altas capacidades, per se, deberían ser un factor más protector que de riesgo por los beneficios que suponen a nivel de procesamiento y, con ello, de adaptación y así parece ser la tendencia en algunos estudios (e.g., Borges del Rosal, Hernández-Jorge y Rodríguez-Naveiras, 2011). Pero se advierte un sesgo habitual en la selección de muestras (habitualmente de alto rendimiento académico) y son muchos los autores 
que advierten del riesgo en la interacción con el medio que puede acabar convirtiendo en vulnerabilidad lo que era potencialidad (e.g., Martin, Burns y Schonlau, 2010) lo que además puede ir variando a lo largo del ciclo vital (Francis, Hawes y Abbott, 2016) pues la vulnerabilidad no es estática ni se manifiesta de las misma forma en las diferentes etapas de la vida. En ese sentido, merece una especial atención la adolescencia, que supone el mayor pico de incidencia de la anorexia nerviosa (e.g., Smink, van Hoeken y Hoek, 2012) e implica cambios físicos, psicológicos, sociales y emocionales que interactúan con las altas capacidades (Moon y Dixon, 2006; Vialle, Heaven y Ciarrochi, 2007).

\section{Asincronías evolutivas}

El término "síndrome de disincronía" fue acuñado por Terrasier (1992) para referirse a la falta de sincronización entre diferentes áreas del desarrollo evolutivo del niño/a con altas capacidades, tanto a nivel interno como externo o social. A nivel interno, se ha focalizado sobre todo en la posible falta de coordinación entre desarrollo cognitivo y nivel afectivo, motriz o del lenguaje. A nivel externo, se podría producir un desfase entre las capacidades del menor y las respuestas por contexto educativo, pares y familia. En la revisión nos centraremos en los aspectos más relacionados con el riesgo de trastornos alimentarios (ver tabla 1).

Tabla 1. Relación entre asincronías y variables de vulnerabilidad a trastornos alimentario.

\begin{tabular}{|c|c|}
\hline Asincronías & Variables de vulnerabilidad a trastornos alimentarios \\
\hline \multirow[t]{2}{*}{ Cognitivo-Emocional } & Baja autoconciencia emocional \\
\hline & Auto-invalidación emocional \\
\hline \multirow[t]{3}{*}{ Menor-Familia } & Invalidación/Invisibilidad emocional \\
\hline & Rol de hiperresponsabilidad \\
\hline & Sobrevaloración de autoconcepto académico \\
\hline \multirow[t]{3}{*}{ Menor -Contexto educativo } & Perfeccionismo reforzado como expectativa habitual \\
\hline & Invisibilidad emocional-Imagen social hiperadaptada \\
\hline & Sesgos y Conflicto de género en mujeres \\
\hline \multirow[t]{4}{*}{ Menor -Iguales } & Dificultades interpersonales por estigma y acoso por iguales \\
\hline & Dificultades interpersonales por complacencia \\
\hline & Dificultades interpersonales por rol de ayudador compulsivo \\
\hline & $\begin{array}{l}\text { Dificultades interpersonales y de identidad por sentimiento } \\
\text { de no pertenencia, ausencia de experiencias de comparti- } \\
\text { ción psicológica con iguales }\end{array}$ \\
\hline
\end{tabular}

\section{Asincronías internas: Asincronía cognitivo-emocional}

De todas las asincronías internas, la que puede aportar más a comprender el origen de un trastorno alimentario es la asincronía entre el desarrollo cognitivo y el afectivo, dado que las dificultades de gestión emocional han sido señaladas como uno de los principales factores de vulnerabilidad de los trastornos alimentarios (e.g., Fairburn et al., 2003, 2008; Meule et al., 2019). Esta asincronía no signi- 
fica que exista una inmadurez emocional, pero sí que la madurez emocional del niño/a, aunque coincida con lo esperado para su edad cronológica, es menor que su nivel intelectual. Es fácil que esta discrepancia cognitivo-emocional se acentúe si el entorno estimula más los puntos fuertes del menor, tanto porque el menor lo busque (correlación activa) como porque el entorno reaccione a la predisposición que observa (correlación reactiva). Todo ello puede resultar en una excesiva hiperracionalización de los procesos emocionales y auto-crítica de las reacciones más emotivas, normales a nivel evolutivo pero desproporcionadas o infantiles desde una perspectiva puramente racional. De hecho, este afrontamiento puede ser reforzado en su medio social: si su entorno se acostumbra a un determinado nivel cognitivo, por un efecto halo acaban generándose expectativas de un funcionamiento hipermaduro también en el ámbito emocional. El resultado puede ser que el niño/a (1) se adapte a esas expectativas ignorando sus propias emociones y sensaciones (la ausencia de conciencia emocional que repetidamente se ha observado en trastornos alimentarios - e.g., Gilboa-Schechtman, Avnon, Zubery y Jeczmien, 2006) o bien (2) reaccione aumentando su respuesta emocional por la vivencia de invalidación, lo que acrecentaría las probabilidades de entrar en un círculo vicioso. Ambas dificultades de gestión emocional, por defecto y por exceso, se encuentran estrechamente relacionadas con los trastornos alimentarios (Hernangómez, 2018).

\section{Asincronías externas: El niño y su familia}

Incluso aunque se permita la conciencia emocional, el chico/a con altas capacidades puede tener dificultades para expresar sus necesidades, pedir ayuda o mostrar sus vulnerabilidades si se acostumbra a no dar problemas, manejarse de forma autónoma y dar una imagen positiva ante los adultos (Peterson, 2009). Esto facilitaría la asignación y asunción de roles de excesiva responsabilidad (Kerr y Multon, 2015), llegando en casos extremos a la inversión de roles en las relaciones paterno-filiales dado el grado de madurez cognitiva percibido en el menor. Este riesgo se acentuaría en los casos de fallecimiento de alguno de los padres, conflicto parental, separación o divorcio, enfermedad o trastorno mental de algún miembro de la familia nuclear. Esta experiencia es repetida en pacientes con trastorno alimentario (e.g., Dallos y Denford, 2008; Ringer y Crittenden, 2007).

Junto a ello, es necesario prestar atención al riesgo de sobrevaloración del área académica en el autoconcepto del menor: tener un buen autoconcepto académico no significa disponer de una buena autoestima y puede constituir una trampa si es el único aspecto que la persona valora de sí misma en detrimento de aspectos socio-emocionales y sin una autoaceptación básica más allá de los logros. Por ello, se debe recordar al chico/a sus cualidades en el ámbito socio-afectivo más allá del área de las competencias y logros asociados a su talento: el déficit subjetivo en ese área puede derivar en compensaciones durante la adolescencia, cuando más necesita la aceptación por iguales, aumentando el riesgo de trastorno alimentario. 


\section{Asincronías externas: El contexto escolar}

Respecto al desajuste en el contexto escolar, destacan tres aspectos en relación al riesgo de trastorno alimentario: el fomento del perfeccionismo y la autoexigencia, la invisibilización del malestar psicológico en los bien adaptados académicamente y los sesgos de género.

Presentar un ritmo de aprendizaje más rápido que el de los compañeros puede hacer que las tareas escolares resulten poco desafiantes, acostumbrándose a un elevado control sobre la materia, resultados brillantes como norma e intolerancia ante cualquier resultado que no sea excelente (Moon, 2009). Por otra parte, no es infrecuente la búsqueda de estimulación adicional a través de actividades extraescolares, lo que puede convertirse en una sobrecarga excesiva a pesar de ser ellos mismos quienes suelen buscarla (e.g., Peterson, 2009) lo que ocurre sobre todo en el perfil más autoexigente y género femenino (Kerr y Multon, 2015).

En el profesorado se observa una tendencia a confundir la adaptación académica con la adaptación emocional, con una clara discrepancia entre las valoraciones externas del profesor y las valoraciones subjetivas del alumnado con altas capacidades (Vialle et al., 2007). El efecto halo, la mayor dificultad para detectar síntomas internalizantes y muy posiblemente una ocultación, consciente o no, por parte del menor pueden estar en el origen de la invisibilidad de su malestar emocional. Pero los trastornos alimentarios en muchas ocasiones aparecen como la manifestación de un malestar emocional que no ha podido comunicarse de otra forma mientras se mantenía una imagen social impecable. Es necesario por tanto estar atentos a la posibilidad de síntomas afectivos silentes, conociendo que la ansiedad y la sensación de aislamiento suelen aumentar según avanzan en la Educación Secundaria (Assouline, Colangelo, Ihrig y Forstadt, 2006).

Entre las primeras explicaciones sobre la relación entre elevada inteligencia y anorexia nerviosa, Detzner y Schmidt (1986) apuntaban al rol de género dado que las niñas con altas capacidades tienden a ocultar con más frecuencia su condición y se encuentran con respuestas más aversivas en su entorno en caso de no ocultarlo (Foust y Booker, 2007). Los sesgos de género se extienden al profesorado (Bianco, Harris, Garrison-Wade y Leech, 2011) y las instituciones (Petersen, 2013), lo que genera conflictos de rol que se asocian con insatisfacción emocional (Kerr y Multon, 2015), corporal e intentos de control del cuerpo a través de diferentes síntomas alimentarios (Lovejoy, 2001). Se necesitarían contextos que ayuden a las adolescentes a concienciarse y afrontar las contradicciones de su rol de género, cuestionar los modelos tradicionales, buscar relaciones de apoyo y explorar modelos de mujer coherentes con sus valores para encontrar su propia solución al conflicto (Choate, 2005).

\section{Asincronías externas: la relación con iguales}

Los riesgos asociados a las altas capacidades en las relaciones con iguales se vinculan directamente con las dificultades interpersonales en adolescencia y edad 
adulta, otro factor de vulnerabilidad básico en trastornos alimentarios (Fairburn et al., 2003, 2008). Clásicamente se ha considerado que el desarrollo cognitivo superior puede derivar en estigma social y/o la sensación marcada de ser diferente en el/la menor, lo que se vincula con baja autoestima y percepciones más negativas de los demás y de las relaciones sociales (Cross, Coleman y Stewart, 1993). El estigma puede aumentar el riesgo de ser víctima de acoso por pares (González-Cabrera, Tourón, León-Mejía y Machimbarrena, 2018; Peterson y Ray, 2006) y ese estresor constituye un factor de riesgo para el desarrollo de un trastorno alimentario (e.g., Copeland, Bulik, Zucker, Wolke, Lereya y Costello, 2015). Se necesita por tanto una especial atención a esta posibilidad en los alumnos con altas capacidades así como contextos educativos que desafíen los estereotipos y favorezcan una valoración positiva de los logros académicos por los iguales (Moon, 2009; Peterson, 2009).

Aunque el miedo al estigma puede llevar a algunos estudiantes, especialmente mujeres (Peterson, 2009), a sentirse en el dilema de elegir entre la excelencia académica o las relaciones con pares (Gross, 1989), es más frecuente que utilicen otras estrategias como la implicación social activa, las conductas de ayuda y la búsqueda de una aceptación independiente de su condición de superdotado/a (e.g., Swiatek y Cross, 2007). En un estudio con población china de altas capacidades, se observó que durante la infancia tendían más a conductas conformistas mientras que en la adolescencia se orientaban a conductas prosociales (Chan, 2005). Sería interesante si este dato se replica en población occidental ya que, curiosamente, en trastornos alimentarios se ha relacionado la anorexia con el patrón de complacencia y el rol de cuidador compulsivo (Ringer y Crittenden, 2007), que en pacientes con altas capacidades podrían constituir estrategias para la aceptación social por iguales reduciendo el sentimiento de ser diferentes.

\section{Impacto de la asincronía con iguales en el desarrollo de la identidad}

El inicio de planteamientos sobre la identidad y la búsqueda de autonomía e intimidad con el otro suele ser más precoz en los niños con altas capacidades (Gross, 1998) y en la construcción del autoconcepto deberá integrarse la experiencia de "ser diferente", lo que en gran parte dependerá de la respuesta que el entorno haya sido capaz de dar a esa diferencia. Cuando es identificada, validada, integrada y se responde sensiblemente a ella, se optimiza la adaptación; en caso negativo, la respuesta del menor tiene más riesgo de ser desadaptativa y centrada en "sentirse diferente” (Alsop, 2003). Por ejemplo, ante fracasos repetidos en integración social el niño puede negarse su deseo de contactar con otros y autodefinirse como una persona solitaria como forma de resolver la disonancia que le genera el desajuste con su entorno (Andronaco, Shute y McLachlan, 2014). La posibilidad de refugiarse en un mundo imaginario rico facilitaría esta opción. Ese sentimiento de diferencia o no pertenencia es central en el origen de los rasgos narcisistas de personalidad (Dimaggio, Petrilli, Fiori y Mancioppi, 2008), asociados con frecuencia al trastorno alimentario (e.g., Gordon y Dombeck, 2010). Si a ello se une una hipervalencia de 
los valores racionales en detrimento de la conciencia emocional, encontraremos un segundo rasgo de la patología narcisista y del trastorno alimentario.

Baudson y Ziemes (2016) aplican a las altas capacidades los modelos de identidad en minorías, desde una posición de confusión, comparación y alienación hasta la aceptación e integración, sugiriendo que la respuesta de "orgullo" de ser diferente podría ocurrir sólo en aquellas personas que han sufrido mayores adversidades, como una forma de compensación. Precisamente otras minorías, como las personas LGTBIQ, son especialmente vulnerables a los trastornos alimentarios por su condición social minoritaria. De hecho, Bayer, Robert-McComb, Clopton y Reich (2017) demuestran que no es sólo el estrés de pertenecer a una minoría (por las experiencias asociadas de discriminación y trauma) sino que incluso personas con elevada tolerancia al estrés manifiestan un sentimiento de vergüenza, potente mediador para explicar la sintomatología alimentaria, que se explicaría por la internalización del modelo social imperante aunque no se comparta.

Es fundamental considerar la influencia del contexto educativo en la vivencia de las asincronías, especialmente en cuanto a la integración de la diversidad y la atención a aspectos socio-emocionales (Eddles-Hirsch, Vialle, McCormick y Rogers, 2012). El contacto entre menores de altas capacidades puede apoyar la construcción de una identidad positiva, por lo que la agrupación parcial en contextos inclusivos tendría sentido no sólo por objetivos académicos sino también desde objetivos socioafectivos (Baudson y Ziemes, 2016), facilitando experiencias gratificantes con iguales que puedan promover un sentido de pertenencia y estados de compartición (Dimaggio Procacci y Semerari, 2002).

\section{Sensibilidad}

La intensidad y sensibilidad emocional han sido descritas con frecuencia como características propias de las personas con altas capacidades, ligadas habitualmente a un desarrollo moral más avanzado y planteamientos existenciales precoces (López, 2007). Su investigación, sin embargo, no resulta sencilla dada la multiplicidad de aproximaciones al objeto de estudio, inicialmente poco integradas entre sí. Así, podríamos diferenciar aquellas perspectivas que se han centrado en el bajo umbral en la entrada de información y fácil activación del sistema nervioso y aquellas que se han centrado en la capacidad metacognitiva. Entre las primeras, se incluirían las sobreexcitabilidades de Dabrowski (1964, 1967, 1970, 1972, citado en Mendaglio y Tillier, 2006), la alta sensibilidad en el procesamiento sensorial de Aron y Aron (1997), y las investigaciones sobre sensibilidad sensorial. Entre las segundas, se encuentra el concepto de alta sensibilidad multifacética de Mendaglio (1995, 2002).

\section{Sensibilidad como bajo umbral y fácil activación del sistema nervioso}

Tanto las sobreexcitabilidades como la alta sensibilidad en el procesamiento sensorial describen peculiaridades del sistema nervioso de origen constitucional (Homberg, Schubert, Asan y Aron, 2016; Mendaglio, 2012) que afectan al procesa- 
miento de estímulos tanto internos como externos (Greven et al., 2019; Mendaglio y Tillier, 2006), si bien la descripción de las sobreexcitabilidades enfatiza más la respuesta resultante (en las áreas psicomotriz, sensual, intelectual, imaginativa y emocional) mientras que la alta sensibilidad enfatiza más los procesos internos: una mayor conciencia de sutilezas ambientales, facilidad para la sobrecarga por exceso de estimulación, mayor profundidad en el procesamiento de información sensorial y mayor reactividad emocional y empatía (Aron, Aron, y Jagiellowicz, 2012). Además, el concepto de sobreexcitabilidades se asocia a la Teoría de la Desintegración Positiva de Dabrowski, en el contexto del estudio de las altas capacidades, mientras que la alta sensibilidad en el procesamiento sensorial se aplica a población general, aunque sus autores apuntan desde su propuesta inicial a que las altas capacidades podrían asociarse a alta sensibilidad.

A nivel empírico, se ha confirmado la relación entre altas capacidades y sobreexcitabilidades especialmente cuando se utiliza la creatividad artística como criterio para detectar las altas capacidades (Mendaglio y Tillier, 2006; Mendaglio, Kettler y Rinn, 2019). Además a nivel de medida las sobreexcitabilidades se solapan con cinco de las facetas del factor apertura a la experiencia (modelo de los cinco grandes), que a su vez se relaciona también con creatividad e inteligencia tanto en población general como con altas capacidades (Vuyk, Krieshok y Kerr, 2016). En cuanto a la alta sensibilidad al procesamiento sensorial, Bridges y Schendan (2019) apuntan a la relación de la creatividad con esta variable, aunque de momento no hay datos comparativos con muestra control concluyentes (Rinn, Mullet, Jett y Nyikos, 2018). En resumen, los datos existentes apuntan a que una inteligencia superior a la media podría facilitar una mayor sensibilidad, lo que es distinto de presuponer una relación unívoca en todos los casos. Dado que la elevada inteligencia es condición necesaria pero no suficiente para la creatividad, y que la relación entre sensibilidad y creatividad parece más clara en las investigaciones existentes, es muy posible que sea la dimensión creativa, y no la intelectual, la que esté relacionada con una mayor sensibilidad. Lamentablemente, la inclusión de la creatividad como criterio necesario en la detección de altas capacidades no es una práctica habitual en investigación, por lo que se precisan más estudios en esa línea para afirmarlo de forma definitiva.

En el ámbito de los trastornos alimentarios, curiosamente la apertura a la experiencia podría considerarse un marcador específico dado que es el único de los Cinco Grandes que no se relaciona habitualmente con psicopatología del clásico eje I (Malouff, Thorsteinsson y Schutte, 2005) y, sin embargo, sí lo hace con trastornos alimentarios (Bollen y Wojciechowski, 2004; Ghaderi y Scott, 2000; MacLaren y Best, 2009). Aunando las propuestas de Ghaderi y Scott (2000) y Aron et al. (2012), una explicación a estos datos es que la sensibilidad implica una susceptibilidad diferencial al medio (Belsky y Pluess, 2009), esto es: la persona sensible no es necesariamente más vulnerable sino sólo más influenciable ante el ambiente, incrementando la vulnerabilidad en interacción con ambientes negativos, 
pero desarrollando un mejor funcionamiento psicosocial que la media en interacción con un ambiente positivo. Así, la sensibilidad refleja una vida emocional compleja y rica que puede contribuir a un buen ajuste, incluso excepcional, y conducir a una apertura a la experiencia cuando se acompaña de un ambiente positivo que facilita la estabilidad emocional y la sociabilidad, pero puede constituir un riesgo incrementado cuando se combina con historias psicosociales aversivas. La bidimensionalidad de la sensibilidad al procesamiento sensorial y sus relaciones con otras medidas psicológicas confirman esa doble faceta (e.g., Greven et al., 2019; Rinn et al., 2018; Smolewska, McCabe y Woody, 2006).

Desde una perspectiva más descriptiva y específica se ha investigado la sensibilidad sensorial con el Perfil Sensorial (Brown, Tollefson, Dunn, Cromwell y Filion, 2001), encontrándose también diferencias significativas en niños con altas capacidades (Gere, Capps, Mitchell y Grubbs, 2009) y en población con trastornos alimentarios (Merwin et al., 2013; Zucker et al., 2013). Gere et al. (2009) sugieren que las consecuencias de la sensibilidad aumentada pueden ser diferentes de un niño a otro, dependiendo de la capacidad para integrar el exceso de información y modular la propia respuesta regulando la excitabilidad que ello genera. Esto nos lleva a referirnos necesariamente a las habilidades metacognitivas.

\section{Sensibilidad como capacidad metacognitiva}

En su estudio de la sensibilidad en altas capacidades, Mendaglio (1995, 2002) propone conceptualizarla como la conciencia de pensamientos, sentimientos y conductas de uno mismo y otros, tanto en la dimensión cognitiva como en la dimensión afectiva, aludiendo así a las habilidades metacognitivas. Además de las variables de autoconciencia y comprensión de mente ajena, sería fundamental incluir las habilidades de regulación dentro del concepto de metacognición (Carcione y Falcone, 2002).

Aunque Mendaglio (2002) da por supuesto que la elevada metacognición sería característica de las personas de altas capacidades, también enfatiza que sería moldeada por el entorno social. De hecho, la investigación ha demostrado que la influencia de la capacidad cognitiva sobre la metacognición se reduce según aumenta la capacidad (Boor-Klip, Cillessen y van Hell, 2014), siendo más bien una condición necesaria, pero no suficiente. Dada además la relación entre metacognición y apego (Carcione y Falcone, 2002), es obvio que necesitamos incorporar variables ambientales de orden socioafectivo para comprender el origen de la capacidad metacognitiva.

A nivel empírico, se ha demostrado la relación entre la alta sensibilidad al procesamiento sensorial y una mayor conciencia de estados internos (Brindle, Moulding, Bakker y Nedeljkovic, 2015), pero también con más dificultades para la aceptación de los mismos y baja confianza en la capacidad para regularlos. Según la interpretación de los mismos investigadores, la sensibilidad elevada a estímulos internos y externos podría llevar a experiencias repetidas de indefensión ante esta- 
dos internos negativos inevitables, lo que impactaría en el uso de las estrategias de regulación emocional por el sentimiento de ineficacia. En la práctica clínica, con frecuencia observamos personas que ponen en marcha infructuosamente estrategias de regulación que "a otros les funcionan", llegando a la atribución interna y estable ("soy yo quien falla"). Posiblemente tomar conciencia de sus diferencias en sensibilidad y facilitar la aceptación y tolerancia emocional, en línea con las terapias de tercera generación, puede facilitar una narrativa diferente sobre sí mismos y una mayor disponibilidad a no desistir en el uso de un abanico más amplio y profundo de habilidades regulatorias. Es necesario recordar que el desarrollo de habilidades metacognitivas puede predecir el pronóstico de la sintomatología alimentaria (Tasca, 2019).

\section{Sensibilidad y Trastornos alimentarios: Diferentes vías de llegada}

En la figura 1 se integran las variables revisadas respecto a sensibilidad incluyendo también aportaciones de la teoría biosocial de Linehan (1993; Crowell, Beauchaine y Linehan, 2009) para explicar uno de los trastornos de la personalidad más frecuentes en trastornos alimentarios (Martinussen et al., 2017). Linehan propone que la vulnerabilidad emocional es una predisposición biológica que implica (1) elevada sensibilidad a los estímulos emocionales, (2) alta intensidad emocional y (3) lento retorno a la línea base, a lo que habría que añadir en algunos casos la facilidad para el paso de emoción a conducta, que no sólo estaría influido por la intensidad emocional sino también por la impulsividad (Crowell et al., 2009). Todo ello entraría en interacción con variables contextuales, adquiriendo especial importancia un ambiente validante o invalidante a nivel emocional.

Siguiendo la figura 1 , en las diferentes fases del procesamiento por la persona sensible pueden encontrarse distintas vías para llegar a un trastorno alimentario. En primer lugar, el exceso de información sensorial necesita ser integrado: en este sentido, Galiana, Muñoz y Beato (2017) han propuesto la posibilidad de un "trastorno alimentario sensorial" relacionando las dificultades de integración sensorial propias del trastorno de procesamiento sensorial con las encontradas en algunos trastornos alimentarios. Posiblemente, esta vía sea el camino preferente en población con trastorno de espectro autista. En segundo lugar, la hipersensibilidad sensorial origina una intensa y frecuente activación emocional que necesitaría ser aceptada, interpretada y nombrada como tal, ya que lo contrario (activación sin lectura emocional) puede predisponer a alteraciones en la imagen corporal (Monteleone et al., 2017). Efectivamente, se ha demostrado la relación entre sensibilidad sensorial y alteraciones en imagen corporal (Zucker et al, 2013). En tercer lugar, la persona necesita estrategias para tolerar y regular esa activación emocional, ya que los síntomas alimentarios cumplen esa función de forma desadaptativa (Merwin et al., 2013). Una de las estrategias de regulación más básicas es precisamentela evitación, y los síntomas alimentarios ayudan a evitar otras experiencias desagradables (Bell, Coulthard y Wildbur, 2017) sustituyéndolas por sensaciones somáticas intensas. 


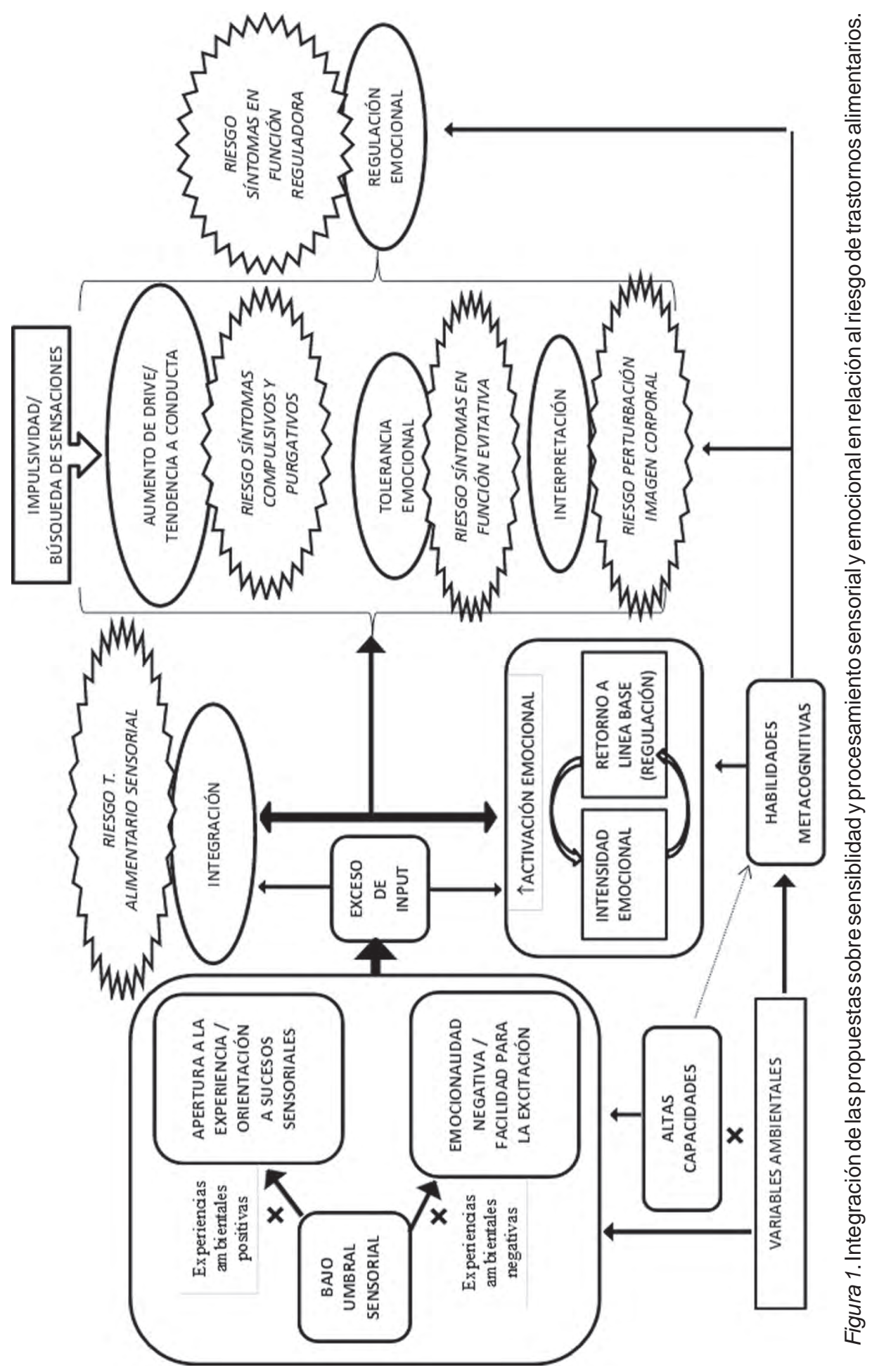


Además, la impulsividad facilitaría, junto con la intensidad emocional, un paso más rápido de la emoción a la conducta, lo que afecta especialmente a la aparición de síntomas compulsivos y purgativos.

La hipersensibilidad a los cambios somáticos haría especialmente complicadas las transiciones vitales como la adolescencia, de forma que los síntomas ayudarían a disminuir esa experiencia sobre todo en ausencia de otras estrategias alternativas más sanas. En sentido contrario, la mejoría en la habilidad para integrar la información sensorial, tolerar la activación emocional, detectar y etiquetar una emoción, y regularla puede disminuir la necesidad de evitar mediante la restricción, la compulsión o la purga.

\section{Perfeccionismo}

El perfeccionismo es una de las variables de vulnerabilidad nucleares en trastornos alimentarios (e.g., Fairburn et al., 2003, 2008; Sassarolli et al., 2008) y un rasgo frecuentemente asociado a las personas con altas capacidades (Stricker, Buecker, Schneider y Preckel, 2019). Sin embargo, si bien se observa una tendencia al perfeccionismo en la población de altas capacidades, el tamaño del efecto indica que el $80 \%$ se solaparía con la población sin altas capacidades, por lo que debemos considerar que no es un rasgo tan característico como se pensaba (íbidem). Ello no le resta importancia, dado que continúa siendo una variable fundamental para explicar dificultades emocionales, adaptativas y de rendimiento académico, pero orienta a la necesidad de analizar los factores que influyen en su origen y centrar en ellos la prevención y detección precoz.

La investigación en perfeccionismo ha demostrado repetidamente la multidimensionalidad de esta variable. Ya en 1991, Hewitt y Flett discriminaron, dentro del perfeccionismo orientado a uno mismo, entre socialmente prescrito (creencia de que los demás sostienen expectativas poco realistas sobre uno mismo) y auto-orientado (expectativas propias poco realistas sobre sí mismo), resultando ambos igualmente daniños, también en niños de altas capacidades (Stornelli, Flett y Hewitt, 2009). Posteriores propuestas se han resumido en dos grandes dimensiones: (1) preocupaciones perfeccionistas, que parecen reflejar una motivación negativa (miedo al fracaso, autocrítica) y (2) esfuerzos perfeccionistas (orientados al logro de metas propias, autoexigencia), que impresionan más asociados a una motivación positiva (búsqueda del placer por el trabajo bien hecho) (Bardone-Cone et al., 2007; Sassaroli et al., 2008). La evidencia empírica apunta a que los esfuerzos perfeccionistas/ autoexigencia son superiores en población de altas capacidades (Stricker et al., 2019) y constituyen un marcador específico de los trastornos alimentarios, frente a las preocupaciones perfeccionistas que compartirían con trastornos habitualmente comórbidos como los trastornos de ansiedad y depresivos (Bardone-Cone et al., 2007; Sassaroli et al., 2008). Obsérvese que los esfuerzos perfeccionistas reflejan una autoexigencia que mantiene la ilusión, en el sentido cognitivo-emocional, de conseguir las metas que se propone, y ello puede implicar un riesgo, como sucede 
en los trastornos alimentarios, en los que parte del trabajo terapéutico se orienta a aceptar la imposibilidad del objetivo de perfección. Precisamente en el ámbito de las altas capacidades, se ha propuesto que un funcionamiento cognitivo superior podría asociarse a mayor capacidad para visualizar objetivos idealizados, pasando fácilmente de la fantasía a la exigencia e intentando resolver la brecha entre realidad e ideales mediante el perfeccionismo (Guignard, Jacquet y Lubart, 2012).

Mientras que el perfeccionismo socialmente prescrito y las preocupaciones perfeccionistas se asocian claramente con disfuncionalidad y psicopatología, los datos sobre perfeccionismo auto-orientado y esfuerzos perfeccionistas/autoexigencia son más inconsistentes. Esta inconsistencia puede responder a que incluye realmente diferentes facetas, sana e insana, según el grado de flexibilidad y los condicionantes que implique para la autoestima el nivel de autoexigencia. Si convive con autoaceptación y tolerancia a los errores, podría identificarse con el denominado "perfeccionismo sano" (e.g., Schuler, 2000) y puede ser un objetivo terapéutico llegar a ese punto. Pero si condiciona la autoestima se caracterizará por la inflexibilidad dado que el incumplimiento de metas supondría una reducción drástica de la autovaloración (Hernangómez, 2018). En este segundo caso, aunque los objetivos fueran auto-impuestos la persona no se auto-regularía realmente por la conciencia de sus necesidades internas y valores sino por una introyección de condicionantes para mantener el autoconcepto (Ryan y Deci, 2009), lo que constituye una de las características de la patología alimentaria (e.g., Carcione, Nicolò y Semerari, 2002).

En cuanto a las vías por las que el perfeccionismo podría afectar de forma diferencial a la población con altas capacidades, se han señalado ya algunas como la capacidad de visualización de objetivos y la ausencia de retos en los primeros años escolares acostumbrándose a la excelencia como resultado habitual. Además, Speirs-Neumeister, Williams y Cross (2009) enfatizan el riesgo de un refuerzo constante basado en etiquetas (e.g., "inteligente"), lo que puede condicionar la autoestima a ese rasgo y facilitar la interpretación de cualquier resultado inferior a lo esperado como el indicador de no ser tan valioso/a como los demás piensan (sensación de fraude), temor que se evitaría con un perfeccionismo compulsivo.

La sensibilidad puede ser también una variable explicativa de los casos en los que se manifiesta el perfeccionismo. Por un lado, una de las variables señaladas en el origen del perfeccionismo ha sido la apertura a la influencia social (SpeirsNeumeister et al., 2009) y precisamente la sensibilidad al procesamiento sensorial ha sido descrita como un factor que aumenta la plasticidad ante el medio. Por otro, la sensibilidad al procesamiento se relaciona con la detección de sutilezas, como pueden ser los errores, y sensibilidad ante las emociones de otros (Aron et al., 2012), lo que puede facilitar el escaneo de errores y la evitación de los mismos en las relaciones por impedir el daño ajeno. Además, a partir de datos empíricos se ha propuesto que la sensibilidad al castigo (BIS), variable relacionada con la sensibilidad al procesamiento (Smolewska et al., 2006), puede encontrarse en el origen del perfeccionismo (Monteleone et al., 2018). 
En cuanto a la prevención del perfeccionismo patológico, se debe evitar especialmente presuponer una buena adaptación psicológica sólo por los resultados académicos: los primeros síntomas del perfeccionista patológico suelen ser internalizantes y su conducta externa acostumbra a ser diligente y complaciente, con dificultades para pedir ayuda, con la consiguiente sorpresa del entorno cuando se manifiestan síntomas más visibles como infrapeso, autolesiones o intentos autolíticos. Es importante facilitar una escucha emocional activa por parte de los adultos de su entorno (Peterson, 2003), prestando especial atención al desarrollo afectivo, reforzando cualidades en esa dimensión y no sólo en la académica, con el objetivo de evitar una sobrevaloración de los logros. Los elogios deberían facilitarse en base a procesos y conductas específicas dado que la etiquetación, sea positiva o negativa, facilita una autoestima condicionada y, por tanto, vulnerable (Kamins y Dweck, 1999). Tanto en el entorno educativo como en el familiar, sería necesario que la población con altas capacidades disponga de un ambiente estimulante en el que desde edades tempranas dispongan de experiencias de pequeños "fracasos" en sus metas para poder ejercitar su capacidad de tolerancia a la frustración y afrontamiento, reinterpretando sus errores como oportunidades para el aprendizaje. Se debe matizar en cualquier caso, dado el sesgo de nuestra sociedad occidental, que una escuela estimulante no es sinónimo de escuela competitiva (Speirs-Neumeister et al., 2009). De hecho, los entornos competitivos pueden promover un perfeccionismo disfuncional, dado que facilitan la comparación social y no la valoración del proceso de aprendizaje y la superación personal. Si el objetivo es evitar el perfeccionismo disfuncional en las aulas, se necesita un mayor énfasis en el proceso y los aprendizajes más que en la ejecución y las calificaciones (e.g., Speirs-Neumeister, 2004).

\section{Conclusiones}

El análisis de las relaciones entre altas capacidades y trastornos alimentarios no resulta fácil en gran parte por motivos metodológicos. La medida de las altas capacidades ha tendido a realizarse de forma reduccionista, mediante medidas de $\mathrm{CI}$ o incluso de rendimiento académico excelente, sesgando claramente las muestras y haciéndolas difícilmente comparables entre sí. Con frecuencia las muestras son pequeñas o falta un grupo control para comparar resultados. Por otra parte, el trastorno alimentario que más se ha investigado ha sido la anorexia nerviosa si bien, dada la migración transdiagnóstica habitual entre los diferentes trastornos alimentarios, es de suponer que los planteamientos aquí desarrollados pueden ser extensibles, como hipótesis, a otros cuadros especialmente si hay antecedentes de anorexia. Además, en el estudio de la anorexia nerviosa debería tenerse en cuenta el grado de infrapeso y la cronicidad, por el efecto de estas variables sobre el rendimiento cognitivo (e.g., Koyama et al, 2012).

En cualquier caso, aunque con prudencia dadas las dificultades señaladas, la evidencia empírica revisada apunta a confirmar que la relación entre altas capacidades y trastornos alimentarios respondería no tanto a características intrínsecas del 
menor sino a la fallida interacción con un medio que no responde ajustadamente a los desafíos que plantea. La persona con altas capacidades se enfrenta a unos retos que no deberían terminar necesariamente en patología. Tanto las asincronías como la sensibilidad o el perfeccionismo pueden orientarse de una forma constructiva y sana. Las altas capacidades constituyen una puerta de entrada a los trastornos alimentarios cuando se encuentran con un contexto poco preparado para responder a sus necesidades. Las familias necesitan apoyo para comprender y atender esas necesidades especiales y las políticas educativas deberían centrarse, además de en la detección, en aportar recursos de formación e intervención que abarquen no sólo la dimensión intelectual sino sobre todo las diferentes facetas creativas y socioafectivas, un cambio que beneficiaría no sólo a esta población específica, sino a toda la población infanto-juvenil.

Algo no estamos haciendo bien a nivel social y educativo cuando convertimos en riesgo lo que en principio es potencialidad. No disponemos en este momento de una cultura que valore la sensibilidad, la creatividad, el esfuerzo, las inquietudes intelectuales, artísticas, existenciales o éticas, y ello supone una pérdida no sólo a nivel individual sino también y sobre todo a nivel social. Trabajemos entre todos para que esto cambie.

\section{Referencias bibliográficas}

Ágh, T., Kovács, G., Supina, D., Pawaskar, M., Herman, B.K., Vokó, Z. y Sheehan, D.V.(2016). Asystematic review of the health-related quality of life and economic burdens of anorexa nervosa, bulimia nervosa, and binge eating disorder. Eating and Weight Disorders 21(3), 353-364. https://doi.org/10.1007/s40519-016-0264-x

Alsop, G. (2003). Asynchrony: Intuitively valid and theoretically reliable. Roeper Review, 25(3), 118-127.https:// doi.org/10.1080/02783190309554213

Andronaco, J. A., Shute, R. y McLachlan, A. (2014). Exploring Asynchrony as a Theoretical Framework for Understanding Giftedness: A Case of Cognitive Dissonance? Roeper Review, 36(4), 264-272. https://doi. org/10.1080/02783193.2014.945218

Aron, E. N. y Aron, A. (1997). Sensory-processing sensitivity and its relation to introversion and emotionality. Journal of Personality and Social Psychology, 73(2), 345-368. https://doi.org/10.1037/0022-3514.73.2.345

Aron, E., Aron, A. y Jagiellowicz, J. (2012). Sensory Processing Sensitivity: A Review in the Light of the Evolution of Biological Responsivity. Personality and Social Psychology Review, 16(3) 262-282. https://doi. org/10.1177\%2F1088868311434213

Assouline, S. G., Colangelo, N., Ihrig, D. y Forstadt, L. (2006). Attributional Choices for Academic Success and Failure by Intellectually Gifted Students. Gifted Child Quarterly, 50(4), 283-294. https://doi. org/10.1177/001698620605000402

Bardone-Cone, A. M., Wonderlich, S. A., Frost, R. O., Bulik, C. M., Mitchell, J. E., Uppala, S. y Simonich, H. (2007). Perfectionism and eating disorders: Current status and future directions. Clinical psychology review, 27(3), 384-405. https://doi.org/10.1016/j.cpr.2006.12.005

Baudson, T. G. y Ziemes, J. F. (2016). The importance of being gifted: Stages of gifted identity development, their correlates and predictors. Gifted and Talented International, 31(1), 19-32. http://dx.doi.org/10.108 $0 / 15332276.2016 .1194675$

Bayer, V., Robert-McComb, J. J., Clopton, J. R. y Reich, D. A. (2017). Investigating the influence of shame, depression, and distress tolerance on the relationship between internalized homophobia and binge eating in lesbian and bisexual women. Eating Behaviors, 24, 39-44. https://doi.org/10.1016/j.eatbeh.2016.12.001 
Bell, K., Coulthard, H. y Wildbur, D. (2017). Self-Disgust within Eating Disordered Groups: Associations with Anxiety, Disgust Sensitivity and Sensory Processing. European Eating Disorders Review, 25, 373-380. https://doi.org/10.1002/erv.2529

Belsky, J. y Pluess, M. (2009). Beyond diathesis stress: Differential susceptibility to environmental influences. Psychological Bulletin, 135(6), 885-908. https://doi.org/10.1037/a0017376

Bianco, M., Harris, B., Garrison-Wade, D. y Leech, N. (2011). Gifted Girls: Gender Bias in Gifted Referrals. Roeper Review, 33(3), 170-181. https://doi.org/10.1080/02783193.2011.580500

Bollen, E. y Wojciechowski, F. L. (2004), Anorexia nervosa subtypes and the big five personality factors. European Eating Disorders Review 12(2), 117-121. https://doi.org/10.1002/erv.551

Boor-Klip, H. J., Cillessen, A. H. N. y van Hell, J. G. (2014). Social Understanding of High-Ability Children in Middle and Late Childhood. Gifted Child Quarterly, 58(4), 259-271. https://doi.org/10.1177/0016986214547634

Borges del Rosal, A., Hernández-Jorge, C. y Rodríguez-Naveiras, E. (2011). Evidencias contra el mito de la inadaptación de las personas con altas capacidades intelectuales. Psicothema, 23(3), 362-367.

Borland, J. H. (2009). Myth 2: The Gifted Constitute 3\% to 5\% of the Population. Moreover, Giftedness Equals High IQ, Which Is a Stable Measure of Aptitude: Spinal Tap Psychometrics in Gifted Education. Gifted Child Quarterly, 53(4), 236-238. https://doi.org/10.1177/0016986209346825

Bridges, D. y Schendan, H.E. (2019). Sensitive individuals are more creative. Personality and Individual Differences, 142, 186-195. https://doi.org/10.1016/j.paid.2018.09.015

Brindle, K., Moulding, R., Bakker, K. y Nedeljkovic, M. (2015). Is the relationship between sensory-processing sensitivity and negative affect mediated by emotional regulation? Australian Journal of Psychology, 67(4), 214-221. https://doi.org/10.1111/ajpy.12084

Brown C., Tollefson, N., Dunn, W., Cromwell, R. y Filion, D. (2001). The adult sensory profile: Measuring patterns of sensory processing. The American Journal of Occupational Therapy, 55, 75-82. https://doi. org/10.5014/ajot.55.1.75

Carcione, A. y Falcone, M. (2002). El concepto de metacognición como constructo clínico fundamental para la psicoterapia. En A. Semerari (Ed.), Psicoterapia cognitiva del paciente grave. Metacognición y relación terapéutica (pp. 23-54). Bilbao, España: DDB.

Carcione, A., Nicolò, G. y Semerari, A. (2002). Déficit de representación de los objetivos. En A. Semerari (Ed.), Psicoterapia cognitiva del paciente grave. Metacognición y relación terapéutica (pp. 147-169). Bilbao, España: DDB.

Chan, D. W. (2005). The structure of social coping among Chinese gifted children and youths in Hong Kong. Journal for the Education of the Gifted, 29(1), 8-29. https://doi.org/10.1177/016235320502900102

Choate, L. H. (2005). Toward a Theoretical Model of Women's Body Image Resilience. Journal of Counseling \& Development, 83(3), 320-330. https://doi.org/10.1002/j.1556-6678.2005.tb00350.x

Copeland, W. E., Bulik, C. M., Zucker, N., Wolke, D., Lereya, S. T. y Costello, E. J. (2015). Does childhood bullying predict eating disorder symptoms? A prospective, longitudinal analysis. International journal of eating disorders, 48(8), 1141-1149. https://doi.org/10.1002/eat.22459

Cross, T. L., Coleman, L. J. y Stewart, R. A. (1993). The social cognition of gifted adolescents: An exploration of the stigma of giftedness paradigm. Roeper Review, 16(1),37-40.https://doi.org/10.1080/02783199309553532

Crowell, S. E., Beauchaine, T. P. y Linehan, M. M. (2009). A biosocial developmental model of borderline personality: Elaborating and extending Linehan's theory. Psychological bulletin, 135(3), 495-510. https://doi.org/10.1037/a0015616

Dallos, R. y Denford, S. (2008). A qualitative exploration of relationship and attachment themes in families with an eating disorder. Clinical Child Psychology and Psychiatry, 13(2), 305-322. https://doi. org/10.1177\%2F1359104507088349

Detzner, M. y Schmidt, M. H. (1986). Are highly gifted children and adolescentes especially susceptible to anorexia nervosa? En K. A. Heller y J. F. Feldhusen (Eds.), Identifying and Nurturing the Gifted. An International Perspective (pp. 149-162). Lewiston, NY: Hans Huber Publishers.

Dimaggio, G., Petrilli, D., Fiori, D. y Mancioppi, S. (2008). El trastorno narcisista de la personalidad. La enfermedad de la "vida grandiosa". En G.Dimaggio y A.Semerari (Eds.), Los trastornos de la personalidad. Modelos y Tratamiento (pp. 221-272). Bilbao, España: DDB.

Dimaggio, G., Procacci, M. y Semerari, A. (2002). Déficit de compartición y pertenencia. En A. Semerari (Ed.), Psicoterapia cognitiva del paciente grave. Metacognición y relación terapéutica (pp. 235-280). Bilbao, España: DDB.

Eddles-Hirsch, K., Vialle, W., McCormick, J. y Rogers, K. (2012). Insiders or outsiders: The role of social context in the peer relations of gifted students. Roeper review, 34(1), 53-62. https://doi.org/10.1080/02783193. 2012.627554 
Fairburn, C. G., Cooper, Z. y Shafran, R. (2003). Cognitive behaviour therapy for eating disorders: a "transdiagnostic” theory and treatment. Behaviour Research and Therapy, 41(5), 509-528. https://doi.org/10.1016/ S0005-7967(02)00088-8

Fairburn, C. G., Cooper, Z. y Shafran, R. (2008). Enhanced cognitive behavior therapy for eating disorders ("CBT-E"): An overview. En C. G. Fairburn (Ed.), Cognitive Behavior Therapy and Eating Disorders (pp. 23-34). Nueva York, NY: Guilford.

Foust, R. C. y Booker, K. (2007). The Social Cognition of Gifted Adolescents. Roeper Review, 29(5), 45-47. https://doi.org/10.1080/02783193.2007.11869224

Francis, R., Hawes, D. J. y Abbott, M. (2016). Intellectual Giftedness and Psychopatheology in Children and Adolescents: A Systematic Literature Review. Exceptional Children, 82(3), 279-302. https://doi. org/10.1177/0014402915598779

Friborg, O., Barlaug, D., Martinussen, M., Rosenvinge, J. H. y Hjemdal, O. (2005). Resilience in relation to personality and intelligence. International Journal of Methods in Psychiatric Research, 14(1), $29-42$. https://doi.org/10.1002/mpr.15

Galiana, A., Muñoz, V. y Beato, L. (2017). Connecting Eating Disorders and Sensory Processing Disorder: A Sensory Eating Disorder Hypothesis. Global Journal of Intellectual \& Developmental Disabilities, 3(4), 1-2.

Galmiche, M., Déchelotte, P., Lambert, G. y Marie Pierre Tavolacci, M. P. (2019). Prevalence of eating disorders over the 2000-2018 period: a systematic literature review. The American Journal of Clinical Nutrition, 109(5), 1402-1413. https://doi.org/10.1093/ajcn/nqy342

Garner, D. (1991). Eating disorders in the gifted adolescent. En M. Bierely y J. Genshaft (Eds.), Understanding the gifted adolescent (pp. 50-64). Nueva York, NY: Teachers College Press.

Gere, D. R., Capps, S. C., Mitchell, D. W. y Grubbs, E. (2009). Sensory sensitivities of gifted children. American Journal of Occupational Therapy, 64, 288-295. https://doi.org/10.5014/ajot.63.3.288

Ghaderi, A. y Scott, B. (2000). The big five and eating disorders: A prospective study in the general population. European Journal of Personality, 14(4), 311-323. https://doi.org/10.1002/1099-0984(200007/08)14:4<311::AIDPER378>3.0.CO;2-8

Gilboa-Schechtman, E., Avnon, L., Zubery, E. y Jeczmien, P. (2006), Emotional processing in eating disorders: specific impairment or general distress related deficiency? Depression and Anxiety, 23(6), 331-339. https:// doi.org/10.1002/da.20163

González-Cabrera, J., Tourón, J., León-Mejía, A. y Machimbarrena, J. M. (2018). Informe ejecutivo del proyecto CIBERAACC. Acoso y ciberacoso en estudiantes de altas capacidades: prevalencia y afectación psicológica. La Rioja: Universidad Internacional de la Rioja (UNIR). Recuperado de: http://www.infocoponline. es/pdf/informeciberaacc.pdf

Gordon, K. H. y Dombeck, J. J. (2010). The associations between two facets of narcissism and eating disorder symptoms. Eating Behaviors, 11(4), 288-292. https://doi.org/10.1016/j.eatbeh.2010.08.004

Greven, C. U., Lionetti, F., Booth, C., Aron, E., Fox, E., Schendan, H. E., ... Homberg, J. (2019). Sensory Processing Sensitivity in the context of Environmental Sensitivity: a critical review and development of research agenda. Neuroscience and Biobehavioral Reviews, 98, 287-305. https://doi.org/10.1016/j.neubiorev.2019.01.009

Gross, M. U. (1989). The pursuit of excellence or the search for intimacy? The forced-choice dilemma of gifted youth. Roeper Review, 11(4), 189-194. https://doi.org/10.1080/02783198909553207

Gross, M. U. (1998). The "me" behind the mask: Intellectually gifted students and the search for identity. Roeper Review, 20(3), 167-174. http://dx.doi.org/10.1080/02783199809553885

Guignard, J. H., Jacquet, A. Y. y Lubart, T. I. (2012). Perfectionism and anxiety: a paradox in intellectual giftedness? PloS one, 7(7), e41043. https://dx.doi.org/10.1371\%2Fjournal.pone.0041043

Hernangómez, L. (2018). Apego y trastornos alimentarios: implicaciones para la intervención psicoterapéutica. Revista de psicoterapia, 29(111), 69-92. https://doi.org/10.33898/rdp.v29i111.264

Hewitt, P. L. y Flett, G. L. (1991). Dimensions of perfectionism in unipolar depression. Journal of abnormal psychology, 100(1), 98-101. https://doi.org/10.1037/0021-843X.100.1.98

Homberg, J. R., Schubert, D., Asan, E. y Aron, E. N. (2016). Sensory processing sensitivity and serotonin gene variance: Insights into mechanisms shaping environmental sensitivity. Neuroscience \& Biobehavioral Reviews, 71, 472-483. https://doi.org/10.1016/j.neubiorev.2016.09.029

Kamins, M. L. y Dweck, C. S. (1999). Person versus process praise and criticism: Implications for contingent selfworth and coping. Developmental psychology, 35(3), 835-847.https://doi.org/10.1037/0012-1649.35.3.835

Kerr, B. A. y Multon, K. D. (2015). The Development of Gender Identity, Gender Roles, and Gender Relations in Gifted Students. Journal of Counseling \& Development, 93(2), 183-191. https://doi.org/10.1002/j.15566676.2015.00194.x 
Koyama, K. I., Asakawa, A., Nakahara, T., Amitani, H., Amitani, M., Saito, M., ... Inui, A. (2012). Intelligence quotient and cognitive functions in severe restricting-type anorexia nervosa before and after weight gain. Nutrition, 28(11-12), 1132-1136. https://doi.org/10.1016/j.nut.2012.03.003

Linehan, M. (1993). Cognitive-behavioral treatment of borderline personality disorder. Nueva York, NY: Guilford Press.

López, C., Stahl, D. y Tchanturia, K. (2010). Estimated intelligence quotient in anorexia nervosa: a systematic review and meta-analysis of the literature. Annals of General Psychiatry, 9(1), 40-50. https://doi. org/10.1186/1744-859X-9-40

López, V.A. (2007). Competencias mentalistas en niños y adolescentes con altas capacidades cognitivas. Implicaciones para el desarrollo socioemocional y la adaptación social (Tesis doctoral. Universidad Autónoma de Madrid, España). Recuperado: https://repositorio.uam.es/handle/10486/1968

Lovejoy, M. (2001). Disturbances in the Social Body: Differences in Body Image and Eating Problems among African American and white Women. Gender \& Society, 15(2), 239-261. https://doi. org/10.1177/089124301015002005

MacLaren, V. V. y Best, L. A. (2009). Female students' disordered eating and the big five personality facets. Eating Behaviors, 10(3), 192-195. https://doi.org/10.1016/j.eatbeh.2009.04.001

Malouff, J. M., Thorsteinsson, E. B. y Schutte, N. S. (2005). The relationship between the five-factor model of personality and symptoms of clinical disorders: a meta-analysis. Journal of Psychopathology and Behavioral Assessment, 27(2), 101-114. https://doi.org/10.1007/s10862-005-5384-y

Martin, L. T., Burns, R. M. y Schonlau, M. (2010). Mental Disorders Among Gifted and Nongifted Youth: A Selected Review of the Epidemiologic Literature. Gifted Child Quarterly, 54(1) 31-41. https://doi. org/10.1177/0016986209352684

Martinussen, M., Friborg, O., Schmierer, P., Kaiser, S., Øvergård, K. T., Neunhoeffer, A. L., ... Rosenvinge, J. H. (2017). The comorbidity of personality disorders in eating disorders: a meta-analysis. Eating and Weight Disorders-Studies on Anorexia, Bulimia and Obesity, 22(2), 201-209. https://doi.org/10.1007/ s40519-016-0345-x

Mendaglio, S. (1995). Sensitivity among gifted persons: A multi-faceted perspective. Roeper Review, 17(3), 169-172. https://doi.org/10.1080/02783199509553652

Mendaglio, S. (2002). Heightened Multifaceted Sensitivity of Gifted Students: Implications for Counseling. Journal of Secondary Gifted Education, 14(2), 72-82. https://doi.org/10.4219/jsge-2003-421

Mendaglio, S. (2012). Overexcitabilities and Giftedness Research: A Call for a Paradigm Shift. Journal for the Education of the Gifted, 35(3), 207-219. https://doi.org/10.1177/0162353212451704

Mendaglio, S. y Tillier, W. (2006). Dabrowski's Theory of Positive Disintegration and Giftedness: Overexcitability Research Findings. Journal for the Education of the Gifted, 30(1), 68-87. https://doi. org/10.1177/016235320603000104

Mendaglio, S., Kettler, T. y Rinn, A. (2019). Psychology of Giftedness and the Theory of Positive Disintegration: A Conversation With Sal Mendaglio. Journal of Advanced Academics, 30(4), 500-507. https://doi. org/10.1177/1932202X19869010

Merwin, R. M., Moskovich, A. A., Wagner, H. R., Ritschel, L. A., Craighead, L. W. y Zucker, N. L. (2013). Emotion regulation difficulties in anorexia nervosa: Relationship to self-perceived sensory sensitivity. Cognition and Emotion, 27(3), 441-452. https://doi.org/10.1080/02699931.2012.719003

Meule, A., Richard, A., Schnepper, R. Reichenberger, J., Georgii, C., Naab, S., ... Blechert, J. (2019). Emotion regulation and emotional eating in anorexia nervosa and bulimia nervosa. Eating Disorders, 17(1), 1-17. https://doi.org/10.1080/10640266.2019.1642036

Monteleone, A. M., Cardi, V., Volpe, U., Fico, G., Ruzzi, V., Pellegrino, F., ... Maj, M. (2018). Attachment and motivational systems: relevance of sensitivity to punishment for eating disorder psychopathology. Psychiatry research, 260, 353-359. https://doi.org/10.1016/j.psychres.2017.12.002

Monteleone, A. M., Castellini, G., Ricca, V., Volpe, U., De Riso, F., Nigro, M., .. Maj, M. (2017). Embodiment mediates the relationship between avoidant attachment and eating disorder psychopathology. European Eating Disorders Review, 25(6), 461-468. https://doi.org/10.1002/erv.2536

Moon, S. M. (2009). Myth 15: High-Ability Students Don't Face Problems and Challenges. Gifted Child Quarterly, 53(4), 274-276. https:/doi.org/10.1177/0016986209346943

Moon, S. M. y Dixon, F. A. (2006). Conceptions of Giftedness in Adolescence. En F. A. Dixon y S. M. Moon (Eds.), The handbook of secondary gifted education (pp. 7-33). Waco, TX: Prufrock Press.

Petersen, J. (2013). Gender differences in identification of gifted youth and in gifted program participation: A meta-analysis. Contemporary Educational Psychology, 38(4), 342-348. https://doi.org/10.1016/j. cedpsych.2013.07.002 
Peterson, J. S. (2003). An Argument for Proactive Attention to Affective Concerns of Gifted Adolescents. Journal of Advanced Academics, 14(2), 62-70. https://doi.org/10.4219/jsge-2003-419

Peterson, J. S. (2009). Myth 17: Gifted and Talented Individuals Do Not Have Unique Social and Emotional Needs. Gifted Child Quarterly, 53(4), 280-282. https://doi.org/10.1177/0016986209346946

Peterson, J. S. y Ray, K. E. (2006). Bullying and the gifted: Victims, perpetrators, prevalence, and effects. Gifted child quarterly, 50(2), 148-168. https://doi.org/10.1177\%2F001698620605000206

Pluess, M. y Belsky, J. (2013). Vantage sensitivity: Individual differences in response to positive experiences. PSychological Bulletin, 139(4), 901-916. https://doi.org/10.1037/a0030196

Ringer, F. y Crittenden, P. M. (2007). Eating disorders and attachment: the effects of hidden family processes on eating disorders. European Eating Disorders Review, 15(2), 119-130. https://doi.org/10.1002/erv.761

Rinn, A. N., Mullet, D. R., Jett, N. y Nyikos, T. (2018). Sensory processing sensitivity among high-ability individuals: A psychometric evaluation of the highly sensitive person scale. Roeper Review, 40(3), 166-175. https://doi.org/10.1080/02783193.2018.1466840

Ryan, R. M. y Deci, E. L. (2009). Promoting self-determined school engagement. En K. R Wentzel y D. B. Miele (Eds.), Handbook of motivation at school (pp. 171-195). Londres: Reino Unido: Routledge.

Sassaroli, S., Lauro, L. J. R., Ruggiero, G. M., Mauri, M. C., Vinai, P. y Frost, R. (2008). Perfectionism in depression, obsessive-compulsive disorder and eating disorders. Behaviour Research and Therapy, 46(6), 757-765. https://doi.org/10.1016/j.brat.2008.02.007

Schuler, P. A. (2000). Perfectionism and Gifted Adolescents. Journal of Secondary Gifted Education, 11(4), 183-196. https://doi.org/10.4219/jsge-2000-629

Smink, F. R. E., van Hoeken, D. y Hoek, H. W. (2012). Epidemiology of Eating Disorders: Incidence, Prevalence and Mortality Rates. Current Psychiatry Reports,14(4), 406-414.https://doi.org/10.1007/s11920-012-0282-y

Smolewska, K. A., McCabe, S. B. y Woody, E. Z. (2006). A psychometric evaluation of the Highly Sensitive Person Scale: The components of sensory-processing sensitivity and their relation to the BIS/BAS and "Big Five". Personality and Individual Differences, 40(6), 1269-1279. https://doi.org/10.1016/j.paid.2005.09.022

Society for the Advancement of Gifted Education (1998). The Emotional Drama of Giftedness. Conference Proceedings of the Annual Conference of the Society for the Advancement of Gifted Education (SAGE) $\left(9^{\text {th }}\right)$. Alberta, Canada: Centre for Gifted Education.

Speirs-Neumeister, K. L., Williams, K. K. y Cross, T. L. (2009). Gifted high-school students' perspectives on the development of perfectionism. Roeper Review,31(4), 198-206. https://doi.org/10.1080/02783190903177564

Speirs-Neumeister, K. L. S. (2004). Understanding the Relationship Between Perfectionism and Achievement Motivation in Gifted College Students. Gifted Child Quarterly, 48(3), 219-231. https://doi. org/10.1177/001698620404800306

Stornelli, D., Flett, G. L. y Hewitt, P. L. (2009). Perfectionism, Achievement, and Affect in Children: A Comparison of Students From Gifted, Arts, and Regular Programs. Canadian Journal of School Psychology, 24(4), 267-283. https://doi.org/10.1177/0829573509342392

Stricker, J., Buecker, S., Schneider, M. y Preckel, F. (2019). Intellectual Giftedness and Multidimensional Perfectionism: a Meta-Analytic Review.Educational Psychology Review. https://doi.org/10.1007/s10648-019-09504-1

Swiatek, M. A. y Cross, T. L. (2007). Construct validity of the social coping questionnaire. Journal for the Education of the Gifted, 30(4), 427-449. https://doi.org/10.4219/jeg-2007-508

Tasca, G. A. (2019). Attachment and eating disorders: a research update. Current Opinion in Psychology, 25, 59-64. https://doi.org/10.1016/j.copsyc.2018.03.003

Terrasier, J. C. (1992). La disincronía. Creatividad y rigidez de la escuela frente al derecho a la diversidad. En Y. Benito (Ed.), Desarrollo y educación de los niños superdotados (pp. 251-260). Salamanca, España: Amarú.

Vialle, W., Heaven, P. C. L. y Ciarrochi, J. (2007). On Being Gifted, but Sad and Misunderstood: Social, emotional, and academic outcomes of gifted students in the Wollongong Youth Study. Educational Research and Evaluation: An International Journal on Theory and Practice, 13(6), 569-586. https://doi. org/10.1080/13803610701786046

Vuyk, M. A., Krieshok, T. S. y Kerr, B. A. (2016). Openness to Experience Rather Than Overexcitabilities: Call It Like It Is. Gifted Child Quarterly, 60(3), 192-211. https://doi.org/10.1177/0016986216645407

Zucker, N. L., Merwin, R. M., Bulik, C. M., Moskovich, A., Wildes, J. E. y Groh, J. (2013). Subjective experience of sensation in anorexia nervosa. Behaviour research and therapy, 51(6), 256-265. https://doi.org/10.1016/j. brat.2013.01.010 